\title{
On the Boundedness and Stability of a Fourth Order Differential Equation $\left({ }^{*}\right)(* *)$.
}

\author{
W. A. SkrapeK - B. S. Lalli (Saskatoon, Canada)
}

Summary. - The aim of this paper is to give sufficient conditions (Theorem 1) for the asymptotic stability (in the large) of the trivial solution $x=0$ of the differential equation

$$
D(x)=x^{(4)}+f(x, \dot{x}, \ddot{x}, \ddot{x})=0 .
$$

$A$ result (Theorem 2) on the boundedness of the solutions of the differential equation $D(x)=$ $=p(t, x, \dot{x}, \ddot{x}, \ddot{x})$ is also established.

\section{1. - Introduction.}

In this paper we investigate the stability and boundedness of the solution of the fourth order differential equation

$$
x^{(4)}+f(x, \dot{x}, \ddot{x}, \ddot{x})=p(t, x, \dot{x}, \ddot{x}, \ddot{x}) .
$$

The results obtained reduce to those which are less restrictive than established by LALLI and SKRAPEK [4] and EzEILo [1] respectively, for the equations

$$
\begin{aligned}
& x^{(1)}+f_{1}(\ddot{x}) \bar{x}+f_{2}(\dot{x}, \ddot{x})+g(\dot{x})+h(x, \dot{x})=p(t), \\
& x^{(4)}+f_{1}(\ddot{x}) \ddot{x}+a_{2}(\ddot{x})+g(\dot{x})+a_{1} x=p(t) .
\end{aligned}
$$

Further they reduce to results which are similar to those obtained by HARRow [3] and Ezeno and TeJumora [2] respectively, for the equations

$$
\begin{aligned}
& x^{(4)}+a \ddot{x}+f_{2}(\ddot{x})+g(\dot{x})+h(x)=p(t), \\
& x^{(4)}+a \ddot{x}+f_{2}(\ddot{x})+g(\dot{x})+h(x)=p(t, x, \dot{x}, \ddot{x}, \ddot{x}) .
\end{aligned}
$$

In what follows, we use the following system which is equivalent to (1.1).

$$
\dot{x}=y, \quad \dot{y}=z, \quad \dot{z}=w, \quad \dot{w}=-f(x, y, z, w)+p(t, x, y, z, w)
$$

(*) Entrata in Redazione il 14 giugno 1977.

(**) The research of the second author was partially supported by N.R.C. grant no. A5293.

1 - Annali di Matematica 
In (1.6), the functions $f$ and $p$ are continuous for all values of their arguments displayed explicitly. The partial derivatives $\partial f / \partial x, \partial f / \partial y, \partial f / \partial z, \partial f / \partial w$ exist and are continuous for al values of their arguments. Also, the existence and uniqueness of the solutions of (1.6) is assumed.

\section{2. - Theorem 1 and Theorem 2.}

Theorem 1. - Let $\left.(\partial / \partial w) f(0,0, z, w)\right|_{w=0}=f_{1}(z)$ and suppose the following conditions hold:

(i) $\quad f(0,0,0,0)=0, f(x, y, 0, w)-f(x, y, 0,0)-f_{1}(0) w=0$

for all $x, y, w$ and there exist finite positive constants $a_{1}, a_{2}, a_{3}$ and finite nonnegative constants $m, n$ such that $f_{1}(z) \geqslant a_{1}$ for all $z$,

$$
\begin{aligned}
& \frac{f(x, y, z, w)+f(0, y, 0,0)-f(x, y, 0,0)-f(0, y, z, 0)}{w} \geqslant a_{1} \\
& \text { for } w \neq 0 \text { and all } x, y, z \text {; } \\
& \frac{f(0, y, z, 0)-f(0, y, 0,0)}{z} \geqslant a_{2} \quad \text { for } z \neq 0 \text { and all } y \text {; } \\
& \frac{f(x, y, z, w)-f(x, y, 0,0)-f_{1}(z) w}{z} \geqslant a_{2}+m \quad \text { for } z \neq 0 \text { and all } x, y, w \\
& \frac{f(0, y, 0,0)}{y} \geqslant a_{3}+n \\
& \text { for } y \neq 0 \text {; }
\end{aligned}
$$

(ii) $[f(x, y, 0,0)-f(0, y, 0,0)] \operatorname{sgn} x>0 \quad$ for $x \neq 0$, and

$[f(x, y, 0,0)-f(0, y, 0,0)] \operatorname{sgn} x \rightarrow+\infty$ as $|x| \rightarrow+\infty ;$

(iii) $(\partial / \partial y)[f(x, y, 0,0)-f(0, y, 0,0)]=0$, and there exist finite positive constants $A_{4}, \Delta_{0}, \Delta_{1}$ and a finite constant $A_{5}$ such that

$$
\begin{array}{ll}
\frac{\partial}{\partial x} f(x, y, 0,0) \leqslant A_{4} \text { and } \frac{\partial}{\partial y}[f(x, y, 0,0)-f(0, y, 0,0)] \leqslant A_{5} & \text { for all } x, y \\
{\left[a_{1} a_{2}-\frac{\partial}{\partial y} f(0, y, 0,0] a_{3}-a_{1} A_{4} f_{1}(z) \geqslant \Delta_{0}\right.} & \text { for all } y, z \\
\frac{4 a_{1} \Delta_{0}}{a_{3}}-\left[A_{4}-\frac{\partial}{\partial x} f(x, y, 0,0)+4 a_{1} A_{5}\right] \geqslant \Delta_{1} & \text { for all } x, y
\end{array}
$$

(iv) $\frac{\partial}{\partial y} f(0, y, 0,0)-\frac{f(0, y, 0,0)}{y} \leqslant \delta_{1}<\frac{2 A_{4} \Delta_{0}}{a_{1} a_{3}^{2}} \quad$ for $y \neq 0$; 
(v) $\left[\frac{1}{z} \int_{0}^{z} f_{1}(s) d s\right]-f_{1}(z) \leqslant \delta_{2}<\frac{2 \Delta_{0}}{a_{1}^{2} a_{3}}$ for $z \neq 0$;

(vi) $(\partial / \partial y)[f(0, y, z, 0) \rightarrow f(0, y, 0,0)] \leqslant 0$ for all $y, z$;

(vii) $[G(x, y, z, w)]^{2} \leqslant\left(4 a_{3} / A_{4}\right) m n$, where

$$
\begin{aligned}
G(x, y, z, w) & =\frac{f(x, y, z, w)-f(x, y, 0,0)-f_{1}(z) w}{z}-a_{2} \\
& -\int_{0}^{x} \frac{1}{y} \frac{\partial}{\partial y}[f(s, y, 0,0)-f(0, y, 0,0)] d s-\frac{a_{3}}{A_{4}} \frac{\partial}{\partial y}[f(x, y, 0,0)-f(0, y, 0,0)]
\end{aligned}
$$

for $y \neq 0, z \neq 0$.

Then every solution of (1.6) with $p(t, x, y, z, w) \equiv 0$ satisfies

$$
x \rightarrow 0, \quad y \rightarrow 0, \quad z \rightarrow 0, \quad w \rightarrow 0 \quad \text { as } \quad t \rightarrow+\infty .
$$

Theorem 2. - Suppose conditions (i), (iii), (iv, (v), (vi), (vii) of Theorem 1 hold and suppose further that

(a) $[f(x, y, 0,0)-f(0, y, 0,0)] \operatorname{sgn} x \rightarrow+\infty$ as $|x| \rightarrow+\infty$;

(b) the function $p(t, x, y, z, w)$ satisfies

$$
|p(t, x, y, z, w)| \leqslant\left\{A_{0}+|y|+|z|+|w|\right\} \varphi(t)
$$

for all $t, x, y, z, w$, where $A_{0}$ is a constant and $\varphi(t) \geqslant 0$ is a continuous function of $t$ such that

$$
\int_{0}^{\infty} \varphi(t) d t<\infty
$$

Then there exists a positive constant $K=K\left(x_{0}, y_{0}, z_{0}, w_{0}\right)$ such that the solution $(x(t), y(t), z(t), w(t))$ of $(1.6)$, determined by the initial conditions

$$
x(0)=x_{0}, \quad y(0)=y_{0}, \quad z(0)=z_{0}, \quad w(0)=w_{0},
$$

satisfies

$$
|x(t)| \leqslant K, \quad|y(t)| \leqslant K, \quad|z(t)| \leqslant K, \quad|w(t)| \leqslant K .
$$

REMARKS.

1) It is easy to verify that the results stated in Theorem 1 and Theorem 2 reduce to results which are slightly less restrictive than those obtained by LALLI and SKRAPEK [4] for equation (1.2). In particular, hypothesis (ii) and (iii) of Theorem 1 
and hypothesis $(a)$ of Theorem 2 are weaker restrictions than the corresponding hypotheses in [4]. The results further reduce to those which are less restrictive than obtained by EzEILo [1] for equation (1.3).

2) Theorem 2 is a slight modification of a result given by Ezeilo and Tejumola in $[2 ;$ Theorem 2].

\section{3. - The function $\mathrm{V}$.}

The proofs of (2.1) and (2.2) depend on properties of the function $V$ defined by

$$
\begin{aligned}
2 V(x, y, z, w) & =2 d_{2} \int_{0}^{x} f(s, y, 0,0) d s-2 d_{2} f(0, y, 0,0) x \\
& +\left(a_{2} d_{2}-A_{4} d_{1}\right) y^{2}+2 \int_{0}^{y} f(0, s, 0,0) d s \\
& +2 d_{1} \int_{0}^{z} f(0, y, s, 0) d s-2 d_{1} f(0, y, 0,0) z \\
& +2 \int_{0}^{z} f_{1}(s) s d s-d_{2} z^{2}+d_{1} w^{2} \\
& +2 f(x, y, 0,0) y-2 f(0, y, 0,0) y+2 d_{1} f(x, y, 0,0) z \\
& +2 d_{2} y \int_{0}^{z} f_{1}(s) d s+2 d_{2} y w+2 z w,
\end{aligned}
$$

where $d_{1}=\varepsilon+1 / a_{1}, d_{2}=A_{4} / a_{3}$ and $\varepsilon$ is a positive constant, the value of which is to be defined to adrantage later.

Now, let

$$
\begin{aligned}
& f_{2}(y, z)=f(0, y, z, 0)-f(0, y, 0,0), \quad h(x, y)=f(x, y, 0,0)-f(0, y, 0,0), \\
& g(y)=f(0, y, 0,0) \quad \text { and } \quad F(z)=\int_{0}^{z} f_{1}(s) d s .
\end{aligned}
$$

Further, let $G_{1}$ and $F_{1}$ be the continuous functions defined by

$$
G_{1}(y)=\left\{\begin{array}{cc}
\frac{g(y)}{y} & y \neq 0 \\
g^{\prime}(0) & y=0
\end{array} \quad F_{1}(Z)=\left\{\begin{array}{cc}
\frac{F(z)}{z} & z \neq 0 \\
f_{1}(0) & z=0 .
\end{array}\right.\right.
$$

The following inequalities can be easily checked.

(A) $\int_{0}^{z} f_{2}(y, s) d s \geqslant a_{2} z^{2}$ for all $y$ and $z$; 
(B) $d_{1}-\frac{f_{1}(z)}{1} \geqslant \varepsilon$ for all $z$,

$d_{1}\left[\frac{f(x, y, z, w)+f(0, y, 0,0)-f(x, y, 0,0)-f(0, y, z, 0)}{w}\right]-1 \geqslant a_{1} \varepsilon$

for $w \neq 0$ and $x, y, z$,

$d_{2}-A_{4} \frac{y}{g(y)} \geqslant 0 \quad$ for $y \neq 0$

(C) $\quad a_{2}-d_{1} g^{\prime}(y)-d_{2} f_{1}(z) \geqslant \frac{\Delta_{0}}{a_{1} a_{3}}-a_{1} a_{2} \varepsilon$ for all $y, z$.

In (3.1), adding and subtracting $2 d_{1} g(y) z$ and applying $(A)$, it is not difficult to verify that

where

$$
\begin{aligned}
2 V(x, y, z, w) & \geqslant U_{1}+U_{2}+U_{3}+\left\{d_{1}-\frac{1}{F_{1}(z)}\right\} w^{2} \\
& +\frac{1}{F_{1}(z)}\left\{w+F(z)+d_{2} y F_{1}(z)\right\}^{2} \\
& +\frac{1}{G_{1}(y)}\left\{h(x, y)+g(y)+d_{1} z G_{1}(y)\right\}^{2}
\end{aligned}
$$

$$
\begin{aligned}
& U_{1}=2 d_{2} \int_{0}^{x} h(s, y) d s-\frac{h^{2}(x, y)}{G_{1}(y)} \\
& U_{2}=\left\{a_{2} d_{2}-A_{4} d_{1}-d_{2}^{2} F_{1}(z)\right\} y^{2}+2 \int_{0}^{y} g(s) d s-y g(y), \\
& U_{3}=\left\{a_{2} d_{1}-d_{2}-d_{1}^{2} \theta_{1}(y)\right\} z^{2}+2 \int_{0}^{z} s f_{1}(s) d s-z F^{\prime}(z) .
\end{aligned}
$$

Now,

$$
\begin{aligned}
U_{1} & \geqslant 2 d_{2} \int_{0}^{x} h(s, y) d s-\frac{h^{2}(x, y)}{a_{3}} \quad \text { by (i) } \\
& =2 \int_{0}^{x}\left[a_{2}-\frac{1}{a_{3}} \frac{\partial}{\partial s} h(s, y)\right] h(s, y) d s \quad \text { since } h(0, y)=0, \\
& \geqslant 0 \text { by (ii) and (iii). }
\end{aligned}
$$

Applying the inequalities $(B)$ and $(C)$ and following the method by Lalli and Skrapek [4] and other it is easily checked that it is possible to select a positive constant $D_{1}$ such that

$$
\begin{aligned}
V(x, y, z, w) & \geqslant D_{1}\left(y^{2}+z^{2}+w^{2}\right) \\
& +\frac{1}{2 G_{1}(y)}\left\{h(x, y)+g(y)+d_{1} z G_{1}(y)\right\}^{2}
\end{aligned}
$$


where $D_{1}$ depends only on the positive constants $a_{1}, a_{2}, a_{3}, A_{4}, \Delta_{0}, \delta_{1}, \delta_{2}$ and a suffciently small $\varepsilon$. Further, $V(0,0,0,0)=0$ and $V(x, y, z, w)$ is positive definite. Also, by (3.5) and (ii) it is clear that $V(x, y, z, w) \rightarrow+\infty$ as $x^{2}+y^{2}+z^{2}+w^{2} \rightarrow+\infty$.

A straight forward calculation yields

$$
\dot{V}=\dot{V}_{1}+\dot{V}_{2}
$$

where

and

$$
\begin{aligned}
\dot{V}_{1}= & -\left[d_{2} g(y) y-\frac{\partial}{\partial x} f(x, y, 0,0) y^{2}\right]-\left[A_{4} d_{1}-d_{1} \frac{\partial}{\partial x} f(x, y, 0,0)\right] y z \\
& -d_{2}\left[f(x, y, z, w)-f(x, y, 0,0)-f_{1}(z) w\right] y \\
& +\left[a_{2} d_{2}+\frac{\partial}{\partial y} h(x, y)\right] y z \\
& -\left[f(x, y, z, w)-f(x, y, 0,0)-f_{1}(z) w\right] z+d_{2} z F(z) \\
& +d_{1} \frac{\partial}{\partial y} f(x, y, 0,0) z^{2}+d_{1} \int_{0}^{z} \frac{\partial}{\partial y} f_{2}(y, s) s d s+d_{2} \int_{0}^{x} \frac{\partial}{\partial y} h(s, y) z d s
\end{aligned}
$$

$$
\dot{V}_{2}=-d_{3}\left[f(x, y, z, w)+f(0, y, 0,0)-f(x, y, 0,0)-f(0, y, z, 0] w+w^{2}\right.
$$

Clearly $\dot{V}_{2}=0$ for $w=0$. For $w \neq 0$,

$$
\begin{aligned}
\dot{V}_{2} & =-\left\{d_{1} \frac{[f(x, y, z, w)+f(0, y, 0,0)-f(x, y, 0,0)-f(0, y, z, 0)]}{w}-1\right\} w^{2} \\
& \leqslant-a_{1} \varepsilon w^{2} \text { by }(B) .
\end{aligned}
$$

For $\dot{V}_{1}$, four cases are considered.

Case 1. $-y=0$ and $z=0$. Then $\dot{V}_{1}=0$.

Case 2. $-y=0$ and $z \neq 0$. Noting that

$$
g^{\prime}(0)=\left.(\partial / \partial y) f(0, y, 0,0)\right|_{y=0}=\left.(\partial / \partial y) f(x, y, 0,0)\right|_{y=0}
$$

by (iii), it follows that

$$
\begin{aligned}
\dot{V}_{1} & =-\left\{\left[\frac{f(x, 0, z, w)-f(x, 0,0,0)-f_{1}(z) w}{z}-d_{1} g^{\prime}(0)\right] z^{2}-d_{2} z F(z)\right\} \\
& +\left.d_{1} \int_{0}^{\infty} \frac{\partial}{\partial y} f_{2}(y, s)\right|_{y=0} s d s
\end{aligned}
$$




$$
\begin{aligned}
& \leqslant-\left\{a_{2}-a_{1} g^{\prime}(0)-d_{2} f_{1}(\theta z)\right\} z^{2} \quad \text { by (i) and (vi) for } 0<\theta<1, \\
& \leqslant-\left[\frac{\Delta_{0}}{a_{1} a_{3}}-a_{1} a_{2} \varepsilon\right] z^{2} \quad \text { by }(C), \\
& \leqslant-\frac{\Delta_{0}}{12 a_{1} a_{8}^{2}} \quad \text { for } \varepsilon>0 \text { sufficiently small. }
\end{aligned}
$$

Case 3. $-z=0$ and $y \neq 0$. In this case

$$
\begin{aligned}
\dot{V}_{1} & =-\left[d_{2} \frac{g(y)}{y}-\frac{\partial}{\partial x} f(x, y, 0,0)\right] y^{2}-d_{2}\left[f(x, y, 0, w)-f(x, y, 0,0)-f_{1}(0) w\right] y, \\
& \leqslant 0 \quad \text { by (i), (iii) and }(B) .
\end{aligned}
$$

Case 4. $-y \neq 0$ and $z \neq 0$. Here it is easily verified that

$$
\begin{aligned}
\dot{V}_{1} & \leqslant-\left[A_{4}-\frac{\partial}{\partial x} f(x, y, 0,0)\right]\left(y+\frac{d_{1}}{2} z\right)^{2}-\left[d_{2} n y^{2}+d_{2} G(x, y, z, w) y z+m z^{2}\right] \\
& -\left\{\left[a_{2}-d_{1} g^{\prime}(y)-\frac{d_{1}^{2}}{4}\left(A_{4}-\frac{\partial}{\partial x} f(x, y, 0,0)\right)-d_{1} \frac{\partial}{\partial y} h(x, y)\right] z^{2}-d_{2} z F^{\prime}(z)\right\} \\
& \leqslant-D_{4}-J_{5},
\end{aligned}
$$

where

$$
\begin{aligned}
U_{4} & =\left[d_{2} n y^{2}+d_{2} G(x, y, z, w) y z+m z^{2}\right], \\
& \geqslant 0,
\end{aligned}
$$

since $[G(x, y, z, w)]^{2} \leqslant 4 m n / d_{2}$ by (vii).

Now

$$
\begin{aligned}
U_{5} & =\left\{\left[a_{2}-d_{1} g^{\prime}(y)-\frac{d_{1}^{2}}{4}\left(A_{4}-\frac{\partial}{\partial x} f(x, y, 0,0)\right)-d_{1} \frac{\partial}{\partial y} h(x, y)\right] z^{2}-z F(z)\right\} \\
& \geqslant\left\{a_{2}-d_{1} g^{\prime}(y)-d_{9} f_{1}(\theta z)-\frac{d_{1}^{2}}{4}\left[A_{4}-\frac{\partial}{\partial x} f(x, y, 0,0)\right]-d_{1} \frac{\partial}{\partial y} h(x, y)\right\} z^{2}
\end{aligned}
$$

for $0<\theta<1$,

$$
\begin{aligned}
& \geqslant\left\{\frac{\Delta_{0}}{a_{1} a_{3}}-a_{1} a_{2} \varepsilon-\frac{d_{1}^{2}}{4}\left[A_{4}-\frac{\partial}{\partial x} f(x, y, 0,0)+\frac{4}{d_{1}} A_{5}\right]\right\} z^{2} \quad \text { by (iii) and }(C), \\
& \geqslant\left\{\frac{\Delta_{0}}{a_{1} a_{3}}-a_{1} a_{2} \varepsilon-\frac{d_{1}^{2}}{4}\left(\frac{4 a_{1} \Delta_{0}}{a_{3}}-\Delta_{1}\right)\right\} z^{2} \quad \text { by (iii), } \\
& =\left\{\frac{d_{1}^{2} \Delta_{1}}{4}-\left(a_{1} a_{2}+\varepsilon \frac{a_{1} \Delta_{0}}{a_{3}}+\frac{2 \Delta_{0}}{a_{3}}\right) \varepsilon\right\} z^{2}, \\
& \geqslant \frac{d_{1}^{2} \Delta_{1}}{8} z^{2} \quad \text { for } \varepsilon>0 \text { sufficiently small } .
\end{aligned}
$$


Therefore, combining all four cases and choosing $\varepsilon>0$ sufficiently small, there exists a positive constant $D_{2}$ such that

$$
\dot{V} \leqslant-D_{2}\left(z^{2}+w^{2}\right)
$$

\section{4. - Proofs of Theorem 1 and Theorem 2.}

The function $V$ is positive definite and satisfies

$$
\nabla(x, y, z, w) \rightarrow+\infty \quad \text { as } \quad x^{2}+y^{2}+z^{2}+w^{2} \rightarrow+\infty
$$

and

$$
\dot{V} \leqslant-D_{2}\left(z^{2}+w^{2}\right),
$$

for any solution $(x(t), y(t), \approx(t), w(t))$ of $(1.6)$. Then the usual Barbashin-type arguments show that

$$
x(t) \rightarrow 0, \quad y(t) \rightarrow 0, \quad z(t) \rightarrow 0, \quad w(t) \rightarrow 0 \quad \text { as } \quad t \rightarrow+\infty .
$$

This completes Theorem 1.

The proof of Theorem 2 is almost identical to that given by Ezeilo and Tejumola [2; Theorem 2]. Therefore only a brief sketch of the proof will be given.

Using the $V$ function (3.1) and noting (3.4) and (3.5) it is easily seen that

$$
\begin{aligned}
V & \geqslant D_{1}\left(y^{2}+z^{2}+w^{2}\right)+d_{2} \int_{0}^{x} h(s, y) d s-\frac{h^{2}(x, y)}{2 G_{1}(y)} \\
& +\frac{1}{2 G_{1}(y)}\left\{h(x, y)+g(y)+d_{1} z G_{1}(y)\right\}^{2} .
\end{aligned}
$$

By ( $a$ ) of Theorem 2 and by continuity of $h(x, y)$ it is clear that a constant $\xi_{0}$ exists such that $h(x, y) \operatorname{sgn} x>0$ for $|x|>\xi_{0}$. It follows that

$$
\begin{aligned}
d_{2} \int_{0}^{x} h(s, y) d s-\frac{h^{2}(x, y)}{2 G_{1}(y)} & \geqslant \frac{1}{2}\left\{2 d_{2} \int_{0}^{x} h(s, y) d s-\frac{h^{2}(x, y)}{a_{3}}\right\}, \\
& =\frac{1}{2} \int_{0}^{x}\left\{d_{2}-\frac{1}{a_{3}} \frac{\partial}{\partial s} h(s, y)\right\} h(s, y) d s, \\
& \geqslant-D_{3},
\end{aligned}
$$

for some positive constant $D_{3}$. Therefore,

$$
V \geqslant D_{1}\left(y_{2}+z^{2}+w^{2}\right)+\frac{1}{2 G_{1}(y)}\left\{h(x, y)+g(y)+d_{1} z G(y)\right\}^{2}-D_{3} .
$$


and moreover by (a) Theorem 2,

$$
V(x, y, z, w) \rightarrow+\infty \quad \text { as } \quad x^{2}+y^{2}+z^{2}+w^{2} \rightarrow+\infty
$$

Also, it is easy to check that

$$
\dot{V} \leqslant-D_{2}\left(z^{2}+w^{2}\right)+D_{4}(|y|+|z|+|w|)|p(t, x, y, z, w)|,
$$

for a positive constant $D_{4}$. By (b) Theorem 2 and since $|y|<1+y^{2}, 2|y||z| \leqslant y^{2}+z^{2}$ it follows that there exist positive constants $D_{5}$ and $D_{6}$ such that

$$
\dot{V} \leqslant D_{5}\left[1+y^{2}+z^{2}+w^{2}\right] \varphi(t),
$$

and further that

$$
\dot{V}-D_{6} V \varphi(t) \leqslant D_{6} \varphi(t)
$$

Therefore,

$$
\nabla(t) \leqslant \psi^{-1}(t)\left\{V(0)+D_{0} \int_{0}^{t} \varphi(s) \psi(s) d s\right\} \quad \text { for } t \geqslant 0,
$$

where $V(0)=V\left(x_{0}, y_{0}, z_{0}, w_{0}\right)$ and $\psi(t)=\exp \left(-D_{6} \int_{0}^{t} \varphi(s) d s\right)$. Since $\psi^{-1}(t)<\infty$ by $(b)$
Theorem 2, it follows from $(4.1)$ and $(4.2)$ that

$$
|x(t)| \leqslant K, \quad|y(t)| \leqslant K, \quad|z(t)|<K, \quad|w(t)| \leqslant K,
$$

for a positive constant $K$.

\section{REFERENCES}

[1] J. O. C. EzEILO, A stability result for solutions of certain fourth order differential equations, J. Math. Anal. Appl., 5 (1962), pp. 136-146.

[2] J. 0. C. Ezeilo - H. O. TEJuMoLA, On the boundedness and the stability properties of solutions of certain fourth order differential equations, Annali di Mat. Pura ed Appl., (IV), 85 (1973), pp. 131-145.

[3] M. HARROW, Further results on the boundedness and the stability of solutions of some differential equations of the fourth order, Siam J. Math. Anal., 1, no. 2 (1970), pp. 189-194.

[4] B. S. LALLI - W. A. SKRAPEK, Further stability and boundedness results for the solutions of some differential equations of the fourth order, Annali di Mat. Pura ed Appl., (IV), 85 (1973), pp. 293-301. 